\title{
CGM Z1.PRO mit CGM Z1.PRO Abrechnungs-Coach - einfach unentbehrlich!
}

\begin{abstract}
Zusätzlich mit dem neuen CGM Z1.PRO Abrechnungs-Coach ausgestattet, ist das neue Zahnarztinformationssystem CGM Z1.PRO noch effizienter geworden. Sicherheit bei der Abrechnung, Zeitersparnis bei den Controlling-Aufgaben und zusätzliche Umsätze durch gezielte Patientenansprache - damit bietet CGM Z1.PRO erhebliche Vorteile gegenüber seinem Vorgänger.
\end{abstract}

CGM Z1.PRO ist ein völlig neues Zahnarztinformationssystem und bietet mit dem CGM Z1.PRO Abrechnungs-Coach eine absolute Ausnahme im Markt! Über den CGM Z1.PRO Abrechnungscoach lässt sich mit nur wenigen Klicks die Quartalsabrechnung perfekt für die abschließende Abrechnungskontrolle vorbereiten. Eine Ergebnisliste zeigt z.B. unberücksichtigte Leistungspositionen oder vergessene/falsch gewählte Begründungen auf. Folge dieses Analyselaufs sind einwandfreie Abrechnungen, die den Zeitaufwand für die Abschluss-Kontrolle wesentlich verkürzen und dem Praxisinhaber die Sicherheit einer lückenlosen Vorbereitung bieten. Bei Live-Tests in Einzelpraxen wurden Abrechnungsdefizite bis zu 8.000 Euro pro Quartal aufgezeigt - mit dem CGM Z1.PRO Abrechnungs-Coach jedoch werden die Abrechnungsdaten schnell und perfekt auf Vollständigkeit und Plausibilität geprüft. Ein Knopfdruck und Abrechnungsverluste gehören der Vergangenheit an! Neben den Performance-Vorteilen sowie der Individualisierbarkeit auf jedes Praxis-Cl und für jeden Arbeitsplatz bietet CGM Z1.PRO mit dem CGM Z1.PRO Abrechnungs-Coach eine Abrechnungssicherheit, die sonst nur eine langjährig auf höchstem Niveau ausgebildete Praxismanagerin bieten kann. Zusätzlich wurden alle Vorgaben für gematik- und Telematikinfrastruktur in der
Softwarearchitektur berücksichtigt; völlig unproblematisch werden neue Betriebssysteme und Hardware-Technologien für CGM Z1.PRO eingestuft.

Darüber hinaus analysiert der CGM Z1.PRO Abrechnungs-Coach perfekt die Potenziale, die sich im Patientenstamm der Praxis verstecken. Mit diesen Informationen kann der Behandler gezielte Angebote zur Verbesserung der Zahngesundheit seiner Patienten machen - und damit signifikant die Patientenzufriedenheit und den Praxisumsatz steigern.

Die überragende Funktionalität der CGM Z1.PRO Features lässt sich direkt im Live-Praxistest demonstrieren. Alle CGM Praxisberater präsentieren gerne das Look-and-Feel von CGM Z1.PRO. In der Live-Demo lässt sich anhand der aktuellen Praxisdaten leicht erkennen, welchen finanziellen Vorteil der Einsatz von CGM Z1. PRO jeder Praxis zu bieten hat.

Einfach bei CGM Dentalsysteme anrufen und einen Demo-Termin vereinbaren. Weitere Informationen unter

www.cgm-dentalsysteme.de oder Tel. 0261-8000 1900.

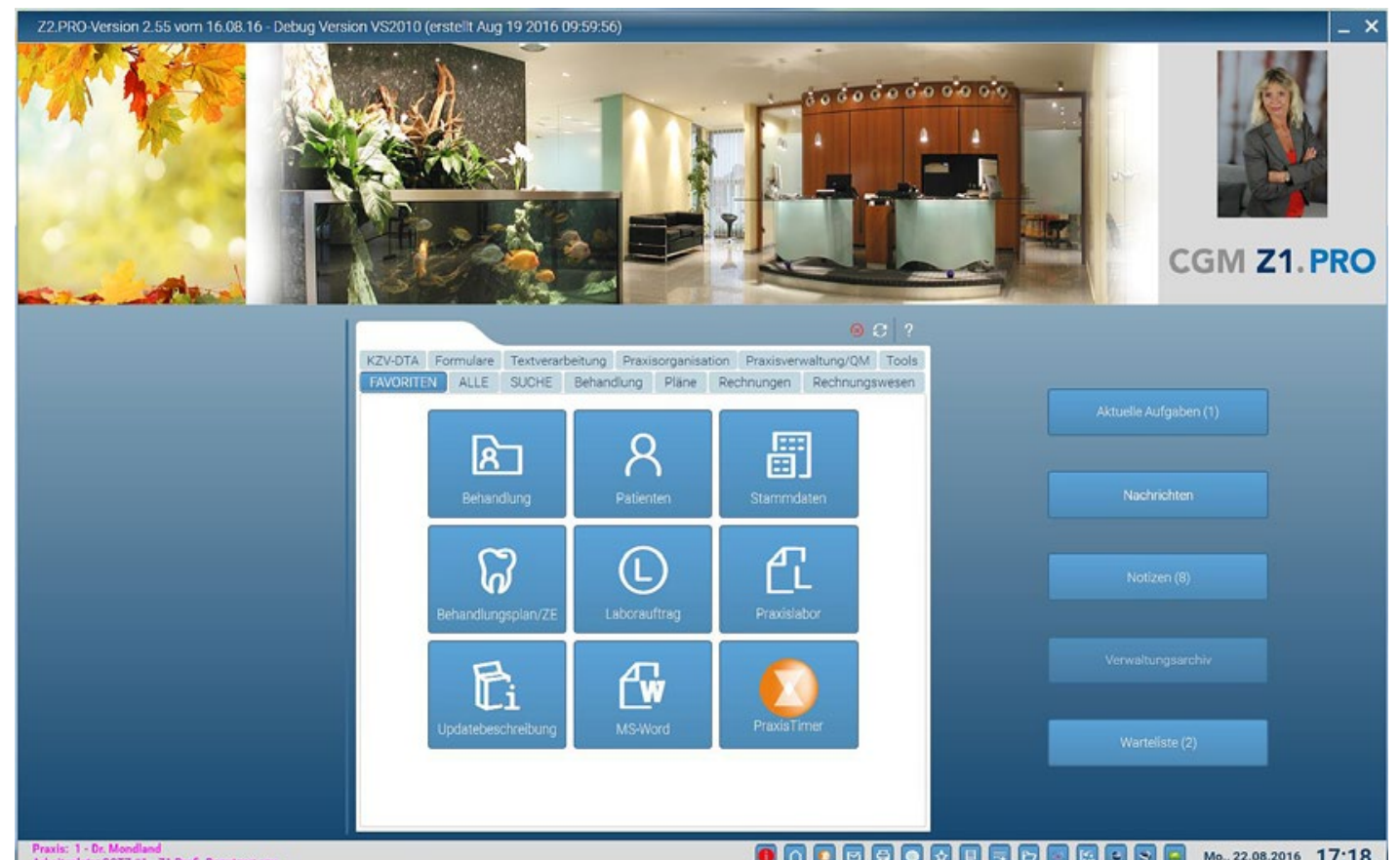

